\title{
Neuronal cultures to study the brain and neurological disorders
}

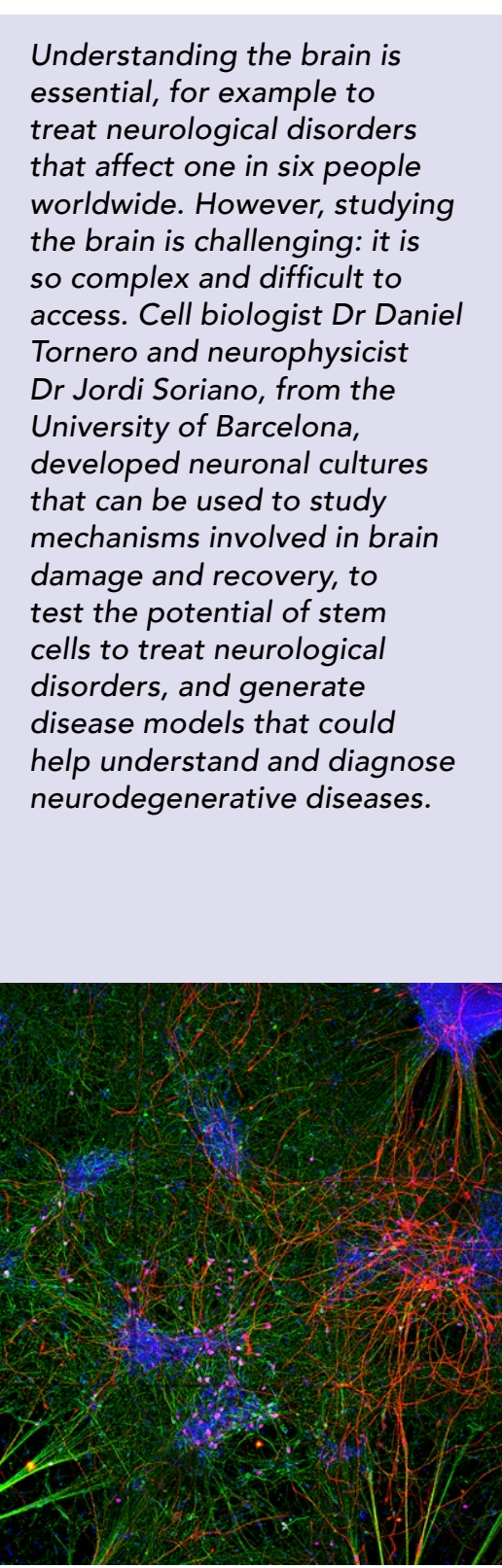

Drs Tornero and Soriano are developing
Reuronal cultures that could help understand and neuronal cultures that could help understand and
diagnose neurodegenerative dissases. Neurons
(green), astrocytes (red) and cell nuclei (blue). he brain is the most complex organ in the human body. Many
mysteries about how it functions we yet to be solved; scientists are still working to understand neurological disorders and to elucidate how the brain develops strategies to either arrest gradual degradation or to repair its complexity and its difficult access, studying the brain represents a big challenge that has evolved towards a truly multidisciplinary endeavour in the last decade.

Neurobiologists, biophysicists and computer simulation experts, amongst others, have joined efforts to understand the brain and also devised strategies to comprehend the building blocks for tis

One of the simplest yet informative cultures. Traditionally two-din are cell cell cultures on Petri dishes (often derived from mouse or rat neurons) and animal models have been used to examine the neurona bechanis involved in health and disease, and to understand aspects as important as network connectivity and neuronal communication. However, 2D models cannot accurately mimic the physiology of the brain in all its complexity. Likewule, all hough animal models are useful tools in many cases, they canno precisely reflect human physiology

Recently, three-dimensional in vitro models combined with the use of stem cells have emerged as a promising At the Univer he secrets of the bran At lead by DrToris neurophysicists, led by Dr Soriano, form a multidisciplinany team that in perfect symbiosis on engineered neuronal cultures.

MIMICKING THE BRAIN IN VITRO In vitro models are more easily accessible than in vivo models, since the number and type of neurons, their interconnectivity, and the size of the neuronal circuit can be tuned. In recent years, new advances in neuroengineering have vastly increased the possibilities of in vitro models. For example, the development of biomaterials for engineered cultures has led to the emergence of systems named 'brain-on-a-chip' or 'brainthe combination of intricate neurona circuit designs with state-of-the-art microfluidics and imaging technologies. These systems establish an in vivolike tissue microenvironment on an engineered platform, guiding cells to behave similarly to those in vivo. This enables, for example, the modelling of a 3D neuronal circuit with organisationa traits similar to the brain. These neuron systems have made it possible to mimic brain's complexity in vitro, and monitor neuronal activity in detail.

One important limitation for the study of human pathologies is the potentia and those originat from neurons used in the labored fy rocents decades, development of stem cellbased technology has opened up cultures with human origin from embryonic stem cells (undifferentiated cells from human embryos that can be grown and expanded in the laborato or by reprogramming somatic cells (any cell in the body other than cells involved in reproduction). After the differentiation of these cells, generated human neurons have the potential to be used disorders. However, before considering disorders. However, before considering them for these purposes, they need and similarity to brain cells in vivo. This can be done using electrophysiological ecordings or by monitoring the changes in calcium levels inside the

\section{CALCIUM IMAGING}

(n) activity is central to quantify the

behaviour of a neuronal circuit and its alterations upon damage. Most neurons communicate through

eurotransmitters, chemical compounds that trigger electrical signals that trave ns and foster the formation of new neurotransmitters at the axonal terminals, passing the electrical signal to other neurons. Two major techniques exist to enregister activity, namely the direct recording of neurons' electrical signt, or the detection of

changes associated

with the release of

neurotransmitters.

For the latter

calcium has been

widely used

since its influx is

tightly related to

means that abrupt information on neuronal activity.

The direct recording of electrical signals gives excellent signal quality but requires the presence of electrodes (or other electric sensors) ext contact wh the neurons, which is a $3 \mathrm{D}$ cutures. On the other hat the 3 culures. On the otherhand, the

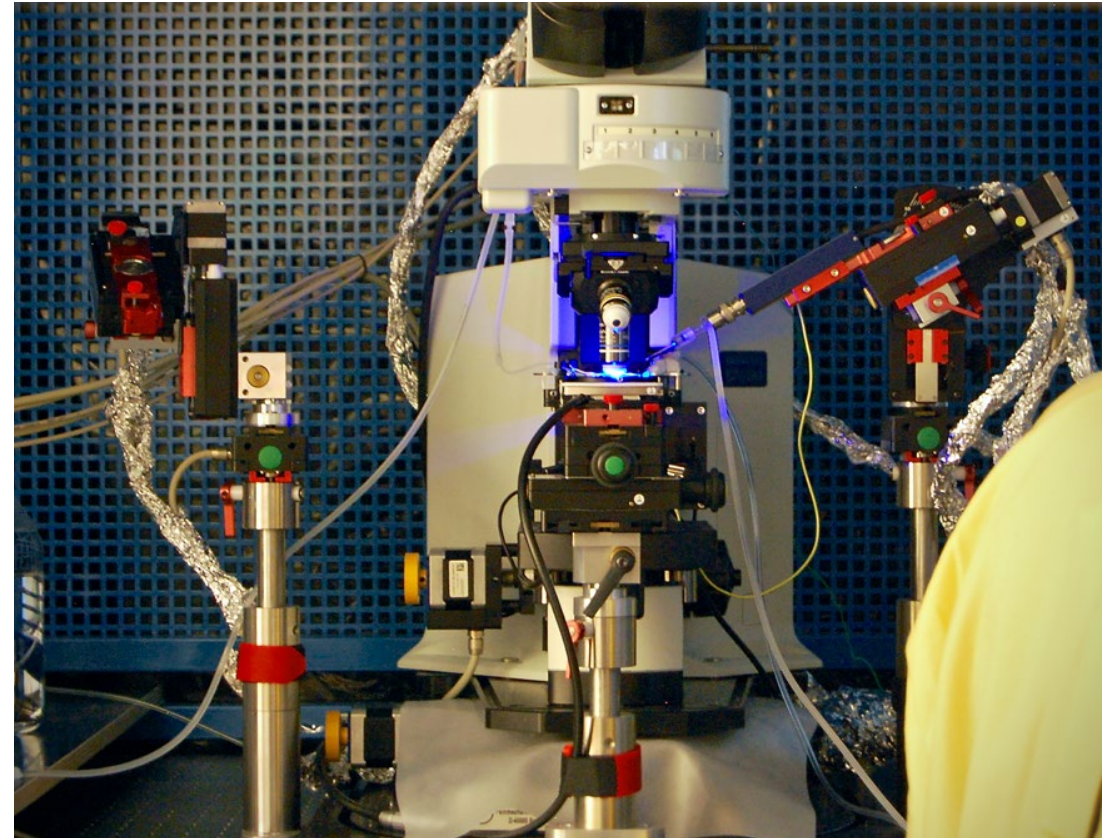

The team use electrophysiology and calcur probe in combination with a highlysensitive imaging device for the detection of fluorescence. This leads to a reduction of signal quality, but it is compensated by a full access to the neurons in the circuit, both in $2 \mathrm{D}$ (fluorescence microscopy) and 3D (lightsheet microscopy

Dr Soriano and Dr Tornero, together with collaborators in photonics, use light-sheet microscopy to observe the

Their approach offers interesting perspectives for modelling network functional alteration and recovery in different situations, from stroke to degenerative disorders.

behaviour of living cells, and the circuits they form, at different time-points and with single cell resolution. More specifically, Dr Tornero and Dr Sorian use these techniques to verify that neurons from their in vitro cultures are functional and similar to cells in vivo. With this information at hand, neurona cultures can be used to understand how the brain works by investigating different circuit designs or by applying
BRAIN DAMAGE AND RECOVERY Two of the brain's major properties self-repair: when damage occurs and alters its function, the brain is able to eorganise its complex networks

Damage can happen in differen example after a head injury or a of (a) maged resulting in functional deficits of diverse extent and severity: the region is unable to sudden activity loss disrupts the neuronal networks in which the damaged brain

region is involved. However, the brain is able to rewire and strengthen the networks, which leads to recovery: partially or totally restored.

Mechanisms involved in the complex functional reorganisation leading to With in vilro approaches, network can be examined in detai. In a ry With in vitro still poorly understood. 


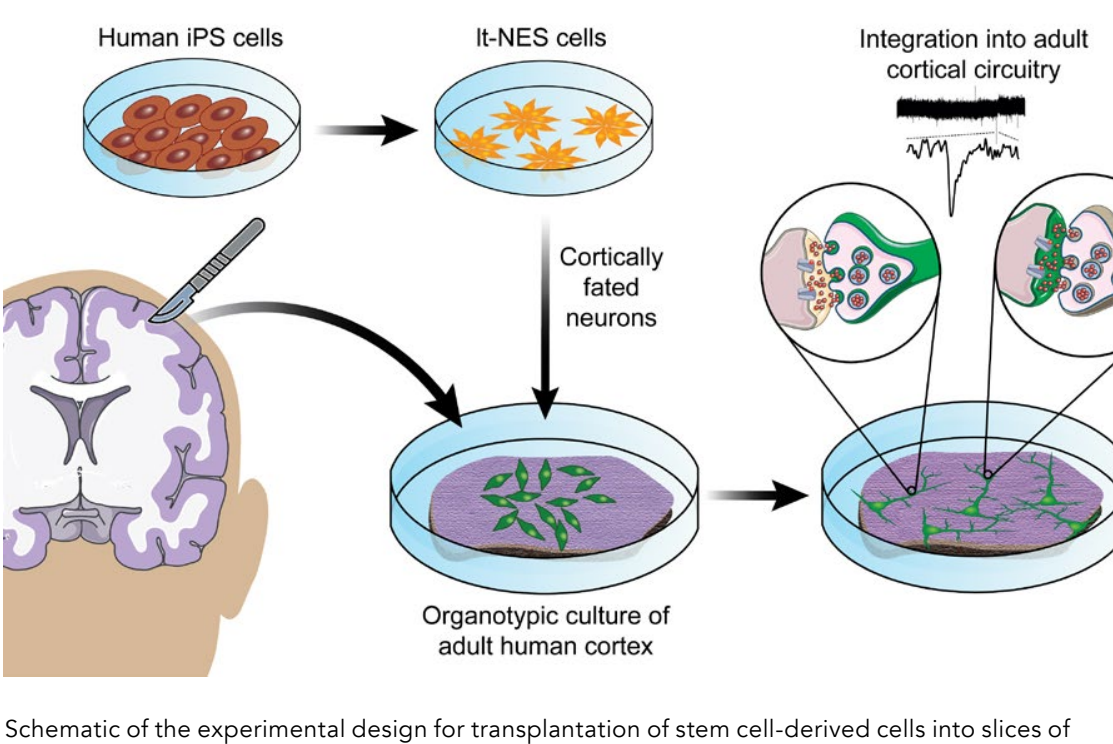

Schematic of the exp
human brain cortex

study, Dr Soriano and Dr Tornero did just that. Using rat neuronal cultures in combination with laser microsurge the researchers studied the complex neuronal network dynamics and its spontaneous recovery upon damage. Laser microsurgery consisted in causing acute damage with a localised laser ablation. In the meantime, calcium fluorescence imaging allowed them to study neuronal activity in the whole network before and after damage. They observed that damage caused by the laser microsurgery led to a Regions adjacent to the lesion col were the most affected, possibly due trit loss connections.

However, activity in

the regions around

the lesion core increased, which allowed. information flow to be quickly rerouted. New functional connections were therefore created while existing ones were strengthened. These mechanisms llowed the network to recover in just 15 minutes, with the network activity back to what it was before damage.

With their in vitro setup, the team from the University of Barcelona showed that restration of the network activity was de by in

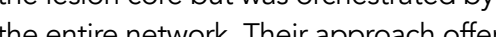
ork activity.

interesting perspectives for modelling network functional loss and recovery degenerative disorders.

\section{NEURODEGENERATIVE DISEASES} during damage and recovery, neuron cultures also offer the possibility to study neurodegenerative diseases, such as Alzheimer's, Parkinson's or Huntington's disease. Affecting more and more people worldwide due to an ageing population and increased life expectancy, these derived from human pluripotent stem cells had always been transplanted into could integrate also into adult human With an engineered $3 \mathrm{D}$ system, Dr Soriano and Dr Tornero examined this possibility They showed that, when implanted onto small slices of adult human cortex maintained in vitro, neurons derived from human pluripoten stem cells could establish functional synaptic connections and integrate in the stablished network. Their results suppor pluripotent stem cells could be used cting the brain.

\section{IN VITRO MODELS OF DISEASE}

Another major part of Dr Tornero neneration of disoase in vitro the in addition to testing whether neurons derived from stem cells can integrate neuronal networks and can therefore be a potential therapeutic approach, they used cells from patients to create disease in vitro models, which can be used to examine the alterations caused by a disease and understand the underlying mechanisms.

More specifically, Dr Soriano and Dr Tornero in collaboration with other aboratories developed in vitro models of a vitro neuronal cultures could be used as disease known as a screening tool to detect early events in Sanfilippo type the generation of the disease and prevent They collected the onset of neurodegeneration. fibroblasts (a type two patients. Using neurons that lead to sensony, motor, and transformed fibroblasts into induced cognitive impairments. pluripotent stem cells, then differentiated A potential therapeutic approach consists of replacing damaged neurons by transplanting stem cells, undifferentiated cells that can proliferate and differentiate into various types of cells. For example, neurons derived from human pluripotent stem cells have the ability to reconnect neuronal networks and improve neurological deficits when rat brain. However, until recely, nerons them into neurons. These neurons presented all the specificities that patients in vivo, with early defects in neuronal activity and altered functional connectivity. Early functional alterations are observed prior to the appearance of the first symptoms, which means that in vitro neuronal cultures could be used as a screening tool to detect the disease earlier and prev reprogramming technologies they
transformed fibroblasts into induced can be observed in neurons from

\section{Behind the Research}

Dr Danie

Tornero

E: daniel.tornero@ub.edu T: +34934034798 W: https://www.ub.edu/porta//

\section{Detail}

Daniel Tornero
C/ Casanova, 143

Faculty of Medicine and Health Science

Universitat de Barcelona

E-08036 Barcelona, Spain

Jordi Soriano

Martí i Franqués 1

Fisica de la Matèria Condensada

Institute of Complex Systems (UBICS)

E-08028 Barcelona, Spain

Bio

Daniel Tornero is an experienced researcher in the field of stem cells. His research is focused on the generation and disease modelling Auman iPSCs for cell therapy Stem Cell Center, he has recently created his own the University of Barcelona.

Jordi Soriano is head of the Neurophysics group focused on the understanding of the interplay activityconnectivity in self-organised and engineered neuron cultures. With more than 15 years of experience, analysis in the context of complex systems (network and information theory and biophysical modelling).

Funding
European

Programme, "La Caixa"

\section{Collaborators}

Isaac Canals (Lund Stem Cell Center, Sweden) - Josep M. Canals (University of Barcelona Spain) - Jordi Alberch (University of Barcelona, Spain) - Pablo Loza (Institute of Photonic Sciences, Barcelona, Spain)

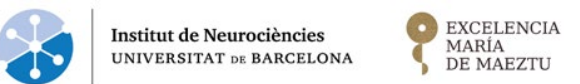

UBIC: $\mid \begin{aligned} & \text { Institute of Complex Systems } \\ & \text { UNIVERSITAA DE BARCLLONA }\end{aligned}$

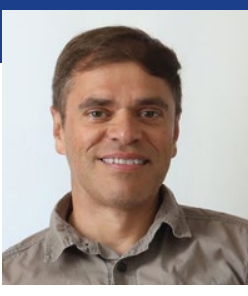

Dr Jordi Soriano

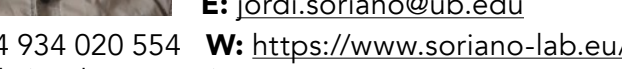
people/jordi-soriano/

References

- Hansen M. G., Tornero D., Canals I., Ahlenius H., \& Kokaia Z. (2019). In Vitro Functional Characterization of Human Neurons and Astrocytes Using Calcium Imaging and
Electrophysiology. Methods in Molecular Biology (Clifto N.J.J., 1819, 73-88. https:///doi.org/10.1007/978-1-4939- To07-8 6 Teller S., Estévez-Priego E., Granell C., Tornero D., Andilla J. Olarte O. E., Loza-Alvarez P., Arenas A., \& Soriano J. (2020). Spontaneous Functional Recovery after Focal Damage in
Neuronal Cultures. eNeuro, 7(1), ENEURO.0254-19.2019. https://doi.org/10.1523/ENEURO. 0254-19.2019 -Gronning Hansen M., Laterza C., Palma-Tortosa S., Kvist G., Monni E., Tsupykov O., Tornero D., Uoshima N., Soriano J., (2020). Grafted humo G., Skibo G., Lindvall O., \& Kokaia Z. neurons integrate into adult human cortical neural circuitry. Advance online publication https//doiorg/10.10021 Advance online
sctm.20-0134

- Canals I., Soriano J., Orlandi J. G., Torrent R., Richaud-Patin Y., Jimenez-Delgado S., Merlin S., Follenzi A., Consiglio A., Vhlageliu L., Grinberg D., \& Raya A. (2015). Activity and Patient-Specific Neuronal Networks. Stem Cell Reports, 5(4), 546-557. https://doi. org/10. 1016/j. stemcr 2015.08.016 - Bang S., Jeong S., Choi N., \& Kim H. N. (2019). Brain-on-a-chip: A history of development and future perspective. Biomicrofluidics, 13(5), 051301. https://doi.
org/10.1063/1.5120555

\section{Personal Response}

What is the next step for your research?

II We will focus our research in the generation of use of precise tissue engineering and gene editing technologies, will be able to mimic human brain pathologies. Monitorisation of calcium intracellular levels will be used to follow the organisational changes maturation. Functionat analysis of the neuronal connectivity in these cultures will allow the study of early events that originate the pathology and the screening of
new therapeutic strategies to revert these defects. 